\title{
IL FANTASTICO DELLA MATERIA: IL TEATRO DELLA RAFFAELLO SANZIO
}

\author{
Mariastella Cassella \\ Universidad de Alcalá \\ mariastellacassella@gmail.com
}

Recibido: 18-07-2014

Aceptado: 12-11-2014

(ㄷ) (1)

\section{SOMMARIO}

La Socìetas Raffaello Sanzio rappresenta, con la sua trentennale esperienza, una delle realtà più innovative del Nuovo Teatro Italiano, avendo sperimentato una scrittura di scena che si è mossa in molteplici direzioni, che vanno dall'azzeramento del testo drammaturgico al suo recupero "problematico» o da un nuovo uso del corpo e dello spazio fino a delineare una diversa concezione di performer e di rapporto con il pubblico. Il nostro tentativo sarà quello di individuare le possibili occorrenze sceniche del fantastico nella prassi del gruppo, strutturando un discorso squisitamente teatrale incentrato sull'uso che se ne fa del corpo e dell'animale e verificando, attraverso alcuni esempi, come entrambi possano funzionare in un'ottica perturbatoria.

Parole chiave: Scrittura scenica, Postorganico, Attore, Corpo, Animale in scena.

\section{Abstract}

The Socìetas Raffaello Sanzio represents one of the most innovative example of the New Italian Theatre. The group has pursued a sophisticated experimentation in performances that have moved in many directions, like a "resetting" of the dramatic text or its problematic recovery, or a new use of body and space to outline a different conception of the relationship between the performer and audience. Our intention is to identify possible occurrences of the fantastic in the group's praxis, structuring a purely theatrical discourse focused on the use of bodies and animals on stage and examining through several examples how both can work in a fantastic mode. 
Keyword: Performance text, Postorganic, Performer, Body, Animals on stage.

Da più di trent'anni il teatro della Raffaello Sanzio lavora, parallelamente, sulla disarticolazione spettacolare e su una destabilizzazione del senso che è, prima di tutto, contrapposizione alla dicotomia tra significante e significato (già esperita, come sappiamo, da molta cultura del Novecento).

È un teatro catastrofico, che si struttura su un'idea di corpo sfregiato mentre subisce, dall'altro lato, il fascino del non umano (sia nella sua accezione animale, come vedremo, che nella trasformazione del personaggio in pupazzo, manichino, congegno meccanico). Romeo Castellucci, Chiara Guidi, Claudia Castellucci e Paolo Guidi hanno dato vita ad una ricerca che, attraverso procedimenti di scarnificazione o, all'opposto, di accumulazione dei significanti, ha intrattenuto relazioni sempre distinte e di non fedeltà con il testo (meglio sarebbe dire i testi) di partenza dello spettacolo.

La grammatica di figure di cui si servono non sembra sancire una volontà di recupero della forza mitica o classica (pensiamo all'Amleto autistico di Amleto o la veemente esteriorità di un mollusco) che queste hanno altrove posseduto, quanto piuttosto di generare uno shock conoscitivo per quella sopravvivenza fantasmatica che le fa permanere, nel ricordo del fruitore, come vestigia di una ormai agonizzante cultura occidentale.

Il recupero delle concrezioni culturali avviene attraverso una ossessione per la forma, da un lato, e per il suo rovesciamento ironico ed anticonvenzionale dall'altro. A funzionare in scena saranno dunque corpi alterati, manomessi, perlustrati e, contemporaneamente, l'animale (inteso come cogenza di un esserci istintuale che si oppone alla razionalizzazione).

Un buon pezzo di teatro deve potersi condensare in un'immagine, che è l'immagine di un organismo, di un animale con quello spirito. Questo animale è una presenza, molto spesso un fantasma, che attraversa la materia, e io con lui. Il problema è essere pellegrini nella materia. La materia è l'ultima realtà. È la realtà finale che ha come estremi il respiro del neo-nato e la carne del cadavere. È un pellegrinaggio che facciamo nella materia. È, quindi, un teatro degli elementi. (Castellucci, 2001: 270-1). 
Le immagini fantastiche di cui si servono, impiantate in riletture sempre originalissime delle opere classiche o in vere e proprie creazioni sceniche, parrebbero rilevare una profonda contraddittorietà che è interna al mondo finzionale che viene descritto, così come una profonda reticenza tanto esplicativa (non veniamo informati delle cause che generano il deragliamento fantastico) quanto semantica (il significato, vuoi simbolico, vuoi allegorico, sembra esserci precluso).

Sarà precisamente attorno ai punti sopra delineati che struttureremo la presente analisi, perlustrando una presenza «materica» del fantastico, un fantastico scenico, diremmo. Oggetto principale d'indagine sarà la famosa riscrittura della trilogia eschilea Orestea (una commedia organica?) del 1995, nella quale si realizza, secondo il nostro avviso, il lavoro più compiuto sul tema fantastico della parabola artistica del gruppo cesenate. L'opera viene deragliata nei territori della fiaba e del fantasmatico, facendone, secondo le parole del regista, «il dramma della putrefazione, dei corpi in decomposizione che tornano in forma di fantasma. In verità sono proprio i morti il motore della storia, gli unici agenti-attori del dramma. E Oreste è il primo personaggio della cultura occidentale percorso dal dubbio, come tutto gli eroi della colpa da Amleto a Lucifero ai personaggi di Dostoevskij. Oreste è il suo braccio sollevato per dare la morte alla madre Clitennestra che si immobilizza nell'aria» (Chinzari, 1995: 12).

Di questi ed altri aspetti ci occuperemo diffusamente, non prima di aver brevemente perlustrato le modalità di reificazione del fantastico generaliter andando poi ad enucleare quei nodi che saranno funzionali al nostro discorso.

Il genere fantastico ha subito, come sappiamo, una forte spinta normalizzatrice a seguito del famoso saggio di Todorov, Introduction à la litterature fantastique, dove lo studioso, rinnovando l'interesse per un genere fino ad allora collocato nella paraletteratura e sicuramente fornendo un'interpretazione restrittiva del genere, faceva notare come il fantastico fosse «l'esitazione provata da un essere il quale conosce soltanto le leggi naturali di fronte ad un avvenimento apparentemente soprannaturale» (Todorov, 1985: 26). Nonostante gli innumerevoli studi che si sono succeduti in seguito, però, lo statuto del fantastico ${ }^{1}$ sembra tutt'oggi essere quello di un fenomeno «indefinibile per definizione» (Albertazzi, 1993: 9).

Ciononostante, potremmo provare ad arginare il pericolo dell'indeterminatezza teorica del genere iniziando col sostenere che il fantastico si costi-

1 Per una sintesi dei risultati delle ricerche sul fantastico si veda anche Scarsella (1986: 201-220). 
tuirebbe come quel luogo nel quale, grazie ad una logica specifica di messa in scena, convivrebbero ciò che è spiegabile con ciò che non lo è (per un suo carattere di assurdo, bizzarro o ignoto) (Solmi, 1978: 126-140). Si accetterebbe, cioè, la presenza di una logica «altra», di una presa di distanza o di una «lievitazione» capace di generare nessi distinti da quanti generati nell'esperienza quotidiana (cfr. Calvino, 1995: 260).

Ritornando a Todorov, è particolarmente interessante la distinzione che quest'ultimo propone con rispetto al meraviglioso e allo strano, soprattutto in una ricerca, come la nostra, che ha per oggetto il teatro. Secondo lo studioso, infatti, il fantastico si situerebbe in una linea di confine: se il fruitore dovesse accettare le leggi di natura che gli vengono proposte si entrerebbe nel meraviglioso, mentre se queste ultime dovessero rimanere intatte ci situeremmo nello strano. Il fenomeno sarebbe, conseguentemente, un genere sempre evanescente.

Del resto, una categoria simile non avrebbe niente di eccezionale. La definizione classica del presente, ad esempio, ce lo descrive come un puro limite tra il passato e il futuro. Il paragone non è gratuito: il meraviglioso corrisponde ad un fenomeno ignoto, ancora mai visto, di là da venire: quindi a un futuro. Nello strano, invece, l'inesplicabile viene ricondotto a fatti noti, a un'esperienza precedente e, di conseguenza, al passato. Quanto al fantastico vero e proprio, l'esitazione che lo caratterizza non può evidentemente situarsi che al presente. (Todorov, 1985: 28)

Il teatro, allora, che è arte del presente, sembrerebbe secondo una lettura di questo tipo il luogo privilegiato per la reificazione del fantastico. Nel caso della nostra ricerca, però, bisognerà tenere conto del valore veritativo che il corpo o l'oggetto possiedono in scena, e che in un certo senso rimanda scopertamente alla realtà e alla cogenza. Vedremo in seguito come questa materialità ontica venga sovvertita nel corso della scrittura scenica messa in marcia dalla Socìetas Raffaello Sanzio.

Proseguiamo la nostra breve ricognizione sul tema del fantastico tenendo presente anche, e soprattutto, la definizione «minima» di Roger Caillois, secondo il quale si genererebbe nella manifestazione di uno «scandalo», una «lacerazione», così come nell'irruzione dell'inammissibile all'interno del paradigma di realtà e dell'inalterabile legalità del quotidiano (2004: 152). Parlare di «scandalo» rimanda alla scoperta vocazione sovversiva ${ }^{2}$ del genere, che fa

2 Si confronti, a riguardo, lo studio di Rosemary Jackson. 
della trasgressione, contemporaneamente, tanto la sua matrice strutturante che la sua funzione fondamentale. Effettivamente, come nota lucidamente Rosalba Campra (2000: 137), è impossibile definire fantastico un testo che non si presenti trasgressivo sia strutturalmente che semanticamente quanto, soprattutto, a livello verbale (come negazione dell'arbitrarietà del segno). Sarà soprattutto questa portata eversiva e trasgressiva, come vedremo, ad interessarci e ad essere particolarmente funzionale alla nostra analisi, in un teatro come quello della Raffaello Sanzio che ha lavorato sistematicamente allo smantellamento della corrispondenza aproblematica tra segno e significato fino alla creazione, come vedremo, di un «teatro iconoclasta». Si tenga però presente, in questa sede, che se la trasgressione della norma rappresenta la caratteristica costituente del genere, dobbiamo anche constatare, con Eco, che nessun mondo narrativo può darsi come totalmente autonomo dal mondo reale. «Un mondo possibile si sovrappone abbondantemente al mondo «reale» dell'enciclopedia del lettore» (2002: 123). Consegue che anche il fantastico dovrà tenere conto delle condizioni «metalinguistiche di costruibilità delle matrici di mondi» (Ibid. 150) organizzando, in maniera eterodossa, le verità logicamente necessarie alla costruzione di una realtà.

Parlando di sovversione, e soprattutto di sovversione linguistica, rimane da chiederci se e quando siano rinvenibili delle operatività comuni o comunque dei principali procedimenti formali. Per Remo Ceserani questi sarebbero ravvisabili nel forte interesse per le capacità "proiettive e creative» che il linguaggio fantastico dovrebbe possedere. «Il mondo fantastico utilizza sino in fondo le potenzialità fantasmatiche del linguaggio, la sua capacità di caricare di valori plastici le parole e formarne una realtà» (1983: 17-18). Il riferimento ad una capacità "plastica» della lingua (che è poi opinione condivisa anche da Diaz Brown, 1996: 110-111) significa che il linguaggio risulta liberato dalla fissità dei procedimenti di scrittura e può, plasmando le proprie regole, «recitare» sé stesso.

Rispetto a quanto siamo andati rilevando fino a questo momento, notiamo come anche il Nuovo Teatro italiano sia venuto mettendo in piedi, sin dalle esperienze delle Cantine Romane o del Convegno di Ivrea 1967, un'opera di smantellamento e smascheramento dell'artificio teatrale ed una risemantizzazione del teatro sul piano dell'evento, facendo della scrittura scenica il suo cardine costituente (cfr. Mango, 2003). Contemporaneamente, le esperienze sovranazionali di Kantor o del più recente Wilson avevano dato luogo ad una contaminazione tra organico (l'attore) ed inorganico (l'oggetto di scena) che non si proponeva più come indagine del binomio animato/inanimato, 
ma, al contrario, come apertura spettacolare all'onirico e al perturbante. Si trattava, cioè, di una prassi squisitamente scenica che traslava l'oggetto reale a contenuti altri, che esulavano dal significato dell'oggetto e lo aprivano al territorio del fantastico, dell'inconoscibile. ${ }^{3}$ Pensiamo alla porta paradossale di Wielopole Wielopole ${ }^{4}$ che, incardinata a delle rotelle, poteva essere spostata a piacimento da un ambiente all'altro di una casa d'infanzia irreale, alla macchina fotografica che diventa mitragliatrice o al letto girevole che consentiva il gioco di scambio fra l'attore e il suo doppio, il manichino. Una riflessione di questo tipo, nonostante la sua brevità, risulterà peculiarmente funzionale alla nostra analisi, essendo il fantastico generato dal teatro della Socìetas Raffaello Sanzio un motore principalmente materico ed inorganico.

Contemporaneamente, si deve tener presente che ha lavorato in teatro come altrove quel progressivo declino dell'accezione normativa di «genere» segnata quasi costitutivamente all'ibridazione, e che ha fatto della trasgressione delle forme e dei canoni un importante veicolo dell'ambiguità. Il postmoderno ha dato avvio, come sappiamo, a quel mondo «ibrido unificato» di cui parla Doležel, in cui entità della finzione possibili ed impossibili convivono nello stesso spazio senza che possa operarvisi una separazione netta e definita (1999: 190). Lo statuto di ambiguità è inoltre accentuato dalla radicale sfiducia in un rapporto trasparente e lineare tra mondo e linguaggio: il linguaggio non può nominare una realtà fluida che solo un pensiero debole (nell'accezione che le dà Vattimo, 1983), nella piena accettazione del gioco linguistico, è capace di generare. La cultura postmoderna sancirebbe, infine, il progressivo sgretolamento della consueta distinzione tra reale e finzionale, riformulando il concetto di rappresentabilità sin dalle sue fondamenta. La progressiva abdicazione di una funzione narrativa forte in favore di un fruitore complice sarebbe il frutto di un radicale rifiuto all'attribuzione di un senso preconfezionato, così come preconizzato da Robbe Grillet nel suo famoso saggio Pour un Nouveau Roman (1965: 50-52).

Come abbiamo già anticipato, la nostra perlustrazione si concentrerà dunque attorno ai paradigmi animali e macchinici/anti-umani presenti nelle prassi sceniche della Socìetas, notando come sia proprio attorno a queste con-

3 Sull'inconoscibilità dell'oggetto teorizzata da Kantor si pensi a quando l'autore dichiara: «L'oggetto mi è sempre interessato. Mi sono reso conto che esso solo è inconoscibile ed inaccessibile» (2000: 76). E ancora: «Il mio punto di partenza è sempre la realtà fisica, un oggetto, ma spesso finisco per approdare nella stessa sfera dell'immaginazione che pertiene ad un'arte che si basa su presupposti metafisici» (2000: 223).

4 Spettacolo storico del regista polacco (1980) nel quale vengono messi in scena e resi protagonisti i meccanismi della memoria, le presenze fantasmatiche e gli incubi che la popolano ossessivamente. 
crezioni d'immagini che si situa tanto la sovversione delle forme quanto il deragliamento verso il fantastico, l'inspiegabile, l'arbitrario, ottenuto attraverso presenze che sembrano sfuggire a qualunque pretesa di leggibilità puntando, pertanto, al caos, al mistero, all'imprevedibile e al sovrannaturale.

Procedendo con ordine, però, riteniamo importante sottolineare come negli spettacoli della Socìetas Raffaello Sanzio il deragliamento del significato agisca già a livello performativo, attraverso l'uso sistematico di presenze che è impossibile far rientrare nella definizione corrente di attore (cfr. Ponte di Pino, 2013: 57): la perlustrazione del limen avviene infatti per difetto o per eccesso di bios. Da una parte fa irruzione l'animale, come portatore del caotico, dall'altra funzionano come reagenti tutta una serie di oggetti meccanici, che dialogano con la materia pulsante del performer facendo deflagrare la messinscena nei territori dello sconosciuto e del mistero.

Iniziando il nostro affondo nella prassi scenica del gruppo, e analizzando l'uso che qui si fa dell'animale, ${ }^{5}$ vedremo come questo funzioni come deriva del naturale nel soprannaturale, nell'inspiegabile e nel fantastico. È fatto noto che quello dell'ensemble cesenate sia un teatro che si serve della realtà cogente della scena per aprirla verso nuovi confini, rifiutando il naturalismo in favore di una superrealtà, ripudiando il linguaggio tradizionale per aprirlo e smantellarlo fino all'afasia (pensiamo alla lingua Generalissima ideata in occasione dello spettacolo Kaputt Necropolis del 1984 o all'autismo del protagonista di Amleto). Sul manifesto offerto agli spettatori in occasione di Santa Sofia. Teatro Khmer (1986) leggiamo, difatti, un inequivocabile:

Questo è il teatro che rifiuta la rappresentazione [...] Questo è il teatro della nuova religione: perciò vieni tu che desideri essere seguace delle colonne dell'Irreale. Il reale lo conosciamo, e ci ha delusi fin dall'età di quattro anni [...] Ma non credere che sia il surrealismo la chiave del problema; la chiave surrealista è completamente sbagliata, nel suo inconscio conservatorismo rielaborato. Questo è il teatro iconoclasta: si tratta di abbattere ogni immagine per aderire alla sola fondamentale realtà: l'Irreale anti-cosmico, tutto l'insieme delle cose non pensate. (Castellucci e Castellucci, 1992: 9)

La nuova super-realtà, che è nelle parole di Castellucci «irreale anticosmico» e l'insieme di quelle concrezioni «ancora non pensate», si produce nello scarto tra ciò che è conosciuto e ciò che non lo è, nella frustrazione delle aspettative spettatoriali, nell'impossibilità di decifrare il senso.

5 Per un'analisi puntuale dell'animalità nella scrittura letteraria, soprattutto del primo Novecento in rapporto ad eventuali modelli antichi, si veda: Marchiori (2010). 
L'animale sarebbe capace, secondo il nostro regista, di generare esattamente ciò: da una parte non può recitare se non sé stesso, offrendosi alla vista senza possedere né offrire alcun filtro interpretativo (come alter-ego dell'attore), dall'altro possiederebbe (vedremo in che senso) una peculiare capacità di aprire lo spettacolo all'ignoto e all'impensato. La lista degli animali impiegati dalla compagnia è lunghissima e non sempre ascrivibile al piano del fantastico; tuttavia la casualità e l'arbitrarietà nell'uso che se ne fa come segno scenico è quantomeno ascrivibile, nel senso che ne dà Todorov e che abbiamo sopra citato, ai territori del meraviglioso e dello strano. L'animale in scena parrebbe rappresentare per sua stessa natura, almeno nell'opinione del nostro regista, lo scacco conoscitivo irrisolvibile dell'istintuale rispetto alla ragione e al paradigma di realtà (l'esperienza liminale del fantastico - unheimlich). Si costituirebbe, cioè, come ponte di contatto con l'alterità rappresentando di per sé il polo oppositivo alla ragione lineare, e capace per questo di scardinare o sospendere la logica della messa in scena per trasferirla in un territorio inesplorato, insieme materico e trasgressivo. Lanimale in scena sembrerebbe dunque, come il fantastico, capace di rivolgere «un monito terribile alla ragione, indicandole i suoi limiti e ricordandole la sua fragilità» (Tortonese, 2002: 181). L'animale come minaccia, come disordine ed ombra fantasmatica dell'attore (dirà Castellucci: «L'animale è senz'altro più efficace, ha una portata più distruttiva», 1995: 170).

Citiamo per esempio, nel pantheon di presenze perturbanti che si annoverano negli spettacoli della compagnia, le numerose apparizione caprine (come il tragos-Agamennone dell'Orestea collegato ad Oreste che, attraverso un complesso sistema di tubi, infonde il proprio alito vitale all'animale, duplicato dall'ariete d'assalto nel Giulio Cesare), i cani randagi di Gilgamesh che ritornano nel terzo atto di Genesi, le molteplici comparse equine o le scimmie Erinni delle Eumenidi.

Il tema animale si declina anche nella sua variante inanimata facendosi volpe imbalsamata (come quella che attraversa il palcoscenico ai piedi di Cassio nel Giulio Cesare), uovo o zampa di gallina (che trasmigrano da un episodio all'altro di Genesi), coniglietto di gesso-Coro nella saga di Oreste o, infine, moltiplicandosi nei numerosi pupazzi coprotagonisti della tragedia di Amleto. I pochi esempi che abbiamo fornito (e che non rendono giustizia all'incredibile presenza quantitativa che il tema possiede) possono già farci comprendere come siano tanto le declinazioni vive che le inanimate a funzionare secondo la logica di un'introduzione di uno spiazzamento ed uno squarcio nella percezione dell'opera, aprendola all'indefinito dell'interpretazione. 
Nella stessa linea espressiva dei corpi alterati (l'Agamennone-down, il Cicerone obeso, l'Antonio laringectomizzato e i Bruto e Cassio interpretati da due ragazze anoressiche nel Giulio Cesare) o del frequente uso dei bambini, l'animale sembra rinviare ad una peculiare concezione del male, così come ad una predilezione per la messa in evidenza del mostruoso, dell'imperfetto e di una creazione divina (pensiamo a Genesi) che sembra produrre unicamente corpi sfregiati ed oltraggiati che condividono con il ferino il caos e la mostruosità.

Quando il tentativo di creazione si fa umano, il risultato sembra non essere migliore, come testimonierebbe l'Atto primo di Genesi dove, nel laboratorio scientifico di Marie Curie, vengono portati a termine degli esperimenti orribili per eguagliare le creazioni tanto del divino come del diabolico. Tutti gli oggetti degli esperimenti sono racchiusi in teche di vetro (altro elemento variamente trasversale nelle messe in scena del gruppo) e vi si annoverano: un pastore tedesco imbalsamato che riproduce una masturbazione macchinica, due ali che battono, private del corpo centrale, ad un ritmo ossessivo e frenetico, due grosse pecore che riproducono una copulazione infinita. Nel finale della scena faranno la loro apparizione una serie di altre teche «tutte riempite con animali impagliati: pecore, montoni, volpi, cani, uccelli, pesci, capre... Tutti stanno a guardare il tentativo di uno di loro di svegliarsi e camminare fuori dalle pareti di vetro della propria conservazione» (Castellucci, 2001: 237).

Quando l'animale non turba la scena con la sua sola presenza di evidenza materica (che si oppone al mondo finzionale della fabula doppiando la fitta ricorrenza di fluidi organici e deiezioni di cui si serve Castellucci, come sperma, sangue, sputi o feci), sembra caricarsi di forti connotazioni fantastiche che alterano la percezione e fanno scivolare lo spettacolo in ambientazioni incomprensibili e terribili. Funzionano in questo senso anche le scimmie Erinni dell'Orestea: lo scivolamento nei confini del fantastico sarebbe generato qui da due operatività critiche complementari. Prima di tutto il lavoro della Raffaello Sanzio svuota il racconto tragico della propria sostanza mitica: il mito non è più ravvivabile come rappresentazione o spiegazione del mondo, sopravvivendovi, di conseguenza, soltanto quanto vi è di anomalo, spaventoso ed inclassificabile. In secondo luogo le scimmie non addomesticate, nella loro natura più selvaggia, fungono da generatrici di un caos centrifugo che invade la scena e problematizza la visione. Le Erinni sarebbero qui la controprova materiale dell'irrazionale, la più pura impossibilità classificatoria che viola e trasgredisce qualsiasi regola o norma. Non sono la figurazione scenica 
delle furie greche, non sono il simbolo o la personificazione della vendetta e in nessun momento potranno trasformarsi in Eumenidi, non essendo altro che sé stesse. Nessun coup de théâtre le farà sparire per sostituirle, ma anzi richiameranno lo stesso Oreste nella gabbia che le contiene. L'inclassificabilità dell'episodio è rafforzata dal lungo sguardo enigmatico che il protagonista rivolge alla sala e allo spettatore, sottolineando la sua natura di discorso oscuro (nell'accezione che ne dà, come vedremo, Caillois). La tragedia dell'Orestea si apre e si chiude nel segno dell'animale: il coro dei vecchi argivi composto da coniglietti meccanici, e quello delle Eumenidi composto da scimmie.

Sono delle scimmie che convivono con gli attori. Rappresentano le Furie, le Erinni che inseguono il matricida Oreste e lo inducono alla follia. Il proscenio a questo punto è stato completamente chiuso da un sipario e c'è soltanto un'apertura che stringe come un diaframma tutta la vicenda in un grande buco. Le Eumenidi si svolge dietro questa apparizione tonda, che è una sorta di lanterna magica dove le figure appaiono e scompaiono come per magia, sempre e comunque filtrate attraverso la visione delle scimmie Erinni, che occupano lo stesso spazio nel quale si trova Oreste, incalzato dal fantasma di Clitennestra e poi avvicinato da Apollo e alla fine da Atena, che risolve la vicenda. (Ponte di Pino, 2013: 61).

La lunga citazione dall'intervista di cui sopra ha il valore di sottolineare come sia anche l'organizzazione spaziale ad essere funzionale al fantastico scenico generato dall'introduzione animale: la visione che si instaura, come un diaframma, duplica quella di una «lanterna magica», donando alle immagini che vi si muovono il carattere di fantasmagoria.

Volendo sintetizzare quanto abbiamo enucleato fino a questo punto, possiamo osservare come il teatro della Raffaello Sanzio nasca e si sviluppi sotto il segno del paradosso: si ispira ai miti pre-tragici, ${ }^{6}$ si professa iconoclasta pur dando luogo a sorprendenti artifici dell'immagine così come spinge la sua ricerca in quel terreno del pre-linguistico che inscena, attraverso l'attore-soma, la propria radicalità contro il linguaggio tradizionale. Amleto, per esempio, si trasforma in un mollusco che regredisce in scena fino all'auto-a-

6 Così Castellucci parlando della condizione pre-tragica, legata all'elemento femminile «Esiste una condizione completamente dimenticata, cancellata, rimossa del teatro occidentale che è quella del teatro pretragico. Ed è rimossa perché è un teatro appunto legato alla materia e allo sgomento della materia. È legato piuttosto a una presenza o a una potenza di tipo femminile, senza dubbio. Capire come il fatto femminile sia (nel mistero della gestazione della vita e nella custodia dei morti) un fatto in realtà che riguarda anche l'espressione artistica, che ha ritrovato in questo termine femminile un rapporto con la vita reale che va dalla nascita alla sepoltura. L'arte nel teatro pretragico aveva questo legame privilegiato con la madre rispetto al corpo generato e al corpo ricomposto per la sepoltura» (2001: 73). 
borto uterino, circondato dal pappagallo Orazio, dal padre orsacchiotto di peluche e da una bambola che ripetutamente imbocca, probabile Ofelia. Non ci sono parole in questo allestimento, che si sviluppa in uno spazio annichilato da teli trasparenti, da valvole che fragorosamente esplodono o dal ronzio dei numerosi impianti elettrici. Il principe di Danimarca è ora un autistico, collocato in uno stato fetale ed escrementizio che non comprende nel proprio regime di senso il potere sanificante della parola, mentre gli elementi fantastici che vi sono contenuti contribuiscono alla deriva dell'interpretazione spettatoriale.

È però nell'Orestea, di cui poco sopra abbiamo analizzato alcuni passaggi, che la riduzione del mito a fiaba fa funzionare i meccanismi di sovversione fantastica in maniera più scoperta. Prima di tutto risulta interessante notare come il corpo degli attori scelti per la tragedia si definisca, ancora una volta, nei termini dell'alterità. Agamennone è un ragazzo down, ${ }^{7}$ Oreste e Pilade due magrissimi efebi vestiti di un solo cappello da clown, Elettra è un'obesa in tutù, così come smisuratamente grassi sono i corpi di una Cassandra rinchiusa in una teca (come gli esperimenti animali di Genesi) e di Clitemnestra. A ciò si aggiunga, come abbiamo accennato, che seguendo la linea estetica che il gruppo è venuto articolando nel corso della sua trentennale carriera, non ci troviamo di fronte ad una canonica messinscena del testo, quanto di fronte ad un materiale che funziona come punto di partenza da tradire, da disarticolare nei suoi presupposti per essere poi rifiutato, reinventato ed alterato.

La collocazione nei territori del fantastico dell'operazione scenica compiuta si spiegherebbe con la piena negazione del mito come nostalgia del sacro e con la messa a morte di qualunque funzione trascendente. L'Orestea svilupperebbe appieno, secondo la nostra lettura, quella disconnessione del senso che se anteriormente sembrava avere a che vedere con una sovranatura determinata e riconoscibile, ora non è più percepibile, in pieno clima postmoderno, come irruzione di un ordine superiore, quanto come perturbamento che si genera sovvertendo le basi dell'ordine prestabilito.

Come nota Rosemary Jackson:

7 Così Castellucci parlando dell'attore, Loris Comandini: «È una cosa a cui pensavo da molto, non una semplice trovata scenica. La presenza di Loris in qualche modo mi attraversa. È contemporaneamente il segno dell'innocenza e il segno della monarchia, del sangue blu: non c'è niente che incarni meglio il senso magistrale del monarca del fatto di essere in qualche modo irraggiungibile. Ma Agamennone è anche la vittima perfetta, designata. Quindi nella semplice presenza di Loris è contenuto questo essere insieme invincibile e vittima perfetta» (Ponte di Pino, 2013: 59). 
Tuttavia mentre le favole e i racconti semireligiosi funzionano attraverso la nostalgia per il sacro, il fantastico moderno rifiuta uno sguardo che guarda all'indietro. Esso è una forma inversa di mito. Si focalizza sull'ignoto del presente, scoprendo il vuoto all'interno di una realtà apparentemente piena. L'assenza stessa è messa in primo piano, collocata al centro semantico del testo. [...] la narrativa moderna aspetta, incessantemente, una epifania impossibile [...] Senza significato, senza trascendenza, il fantastico moderno funziona come se il significato e la trascendenza dovessero essere ricercati. Esso scopre la semplice assenza e il vuoto. Tuttavia continua la sua ricerca per qualcosa di assoluto. (1986: 157)

Innestando porzioni sceniche fantastiche dotate di una profonda ambiguità, la scrittura spettacolare di Castellucci sembra da una parte dialogare con l'elevata oscurità testuale dell'Orestea, e dall'altra sovvertire dall'interno e scopertamente i suoi principi. Come discorso oscuro qui coesisterebbero il senso dell'ineffabile ed una accentuata densità semantica, ambedue capaci di aprire l'opera ad una molteplicità di letture proprio in quanto parrebbero impedirle (Caillois, 2004: 43-44).

Quella della Raffaello Sanzio è, insomma, una costruzione spettacolare basata sulla reticenza, secondo la suggestiva visione che ci offre Prandi (1990: 235) quando sostiene che «interpretare una reticenza non significa reintegrare un segmento represso ma, al contrario, congetturare un messaggio direttamente sulla base di un vuoto irreversibile di contenuto, di un silenzio assoluto».

Come molti racconti fantastici del Novecento, il teatro di Castellucci entra in collisione, dunque, con la sintassi del paradigma di realtà (secondo l'accezione che ne dà la Jackson) più che con la semantica. Non si immettono, cioè, elementi aggiuntivi o avulsi all'interno del paradigma ma si gioca con gli elementi che si hanno a disposizione fino a farne saltare, però, le connessioni logiche. Funzionerebbe qui ciò che Derrida notava a proposito del teatro della crudeltà di Antonin Artaud che non sarebbe una «rappresentazione» quanto «la vita stessa in ciò che ha di irrappresentabile» (1971: 301).

Il fantastico come "confine dell'Ignoto» sul quale si costituiscono le ricerche di Castellucci e i suoi ha per bersaglio, dunque, quella logica classica ed aristotelica che è venuta costituendo l'impalcatura del pensiero occidentale, allineata attorno al principio di identità e non contraddizione così come sulle nozioni di tempo, spazio e relazione.

Con rispetto all'evanescenza di cui parliamo occorre precisare, con Todorov, che è il fantastico stesso ad avere carattere di precarietà, quasi come se fosse ravvisabile compiutamente solo come un segno presente in brani o in 
momenti di opere più che poter costituire un genere definitivo. È per questa sua innata natura di non permanenza che si scivolerebbe nello strano e nel meraviglioso, essendo il primo il racconto di avvenimenti non sovrannaturali bensì incredibili e il secondo la costruzione di un mondo di fiaba accettato dal fruitore. Ricordiamo anche che è attorno a questo nodo che si è centrata quasi unanimemente tutta la critica posteriore, che ha visto nel rigore eccessivo il sacrificio di una pluralità di occorrenze forzate da una ripartizione teorica troppo angusta. ${ }^{8}$ Ciò che tentiamo di mostrare è, ciononostante, come l'irruzione dell'elemento fantastico nel teatro della Raffaello Sanzio funzioni generando, attraverso l'irruzione dell'elemento perturbante, una sua non accettazione e spiegazione razionalizzante. L'elemento fantastico funziona come destabilizzazione del racconto e della scena, e sembra perseguire quella stessa funzione trasgressiva nei confronti dei tabù sociali che già l'Ottocento letterario aveva praticato per rappresentare quelle perversioni che si impediva di affrontare scopertamente. Il fantastico funziona qui, dunque, come generatore di inquietudine, straniamento, dubbio e di non adeguatezza della soluzione prospettata, mettendo in crisi la nostra fiducia nei meccanismi conoscitivi ed interpretativi (cfr. Lugnani, 1983: 57).

Proseguendo la nostra perlustrazione, ricorderemo quanto abbiamo accennato circa l'esposizione di attori le cui caratteristiche fisiche peculiari inneschino il senso attraverso segni particolarissimi che ne definiscono incontrovertibilmente l'esteriorità, facendone figure più che personaggi. Accade nell'Orestea, ad esempio, che Apollo sia veramente un uomo senza braccia, sovrapponendosi all'immagine statuaria della divinità pur mantenendo, nel corso della rappresentazione, un'aura misterica indubitabile. Se l'animale marcava il confine tra bestialità e razionalità, l'insieme di costruzioni macchiniche che pullulano nel teatro della compagnia giocano sul confine tra animato ed inanimato e sulla riduzione del corpo del performer ad oggetto, pratica già esemplificata dalla cosalità dello stare in scena della corporatura alterata (e di cui abbiamo fornito qualche, seppur sparuto, esempio).

La replicabilità dei corpi (a cui abbiamo avuto occasione di accennare riguardo alla materia ferina), così come il corpo artificiale o l'innesto robotico, rappresenta una vera e propria sfida allo sguardo ermeneutico, determinando la medesima incapacità cognitiva che si esperisce rispetto al «doppio». La

8 Effettivamente la trattazione dello studioso, sacrificando la specificità dei racconti a esigenze di esaustività e simmetria, perde di vista in più occasioni il suo reale oggetto d'indagine. Emblematica è l'esclusione della pressoché totale produzione di Poe dal genere fantastico, e la sua attribuzione alla categoria dello «strano». 
visione e l'esposizione di corpi alterati o direttamente clonati opera, infatti, nel paradosso, generando una divaricazione insostenibile e mostruosa tra apparenza ed essenza. L'immaginario cyber della contemporaneità, come sappiamo, lavora sul corpo invadendolo attraverso la tecnologia: in una concezione contemporanea che ne fa sempre più uno «strumento» (e come tale modificabile a piacimento) il corpo diviene il ricettacolo preferenziale all'innesto di pezzi altri (che possono essere variamente clonati, trapiantati, costruiti). Il lavoro sull'artificialità impiantata sulla/nella materia organica non solo è componente comune a molta letteratura cyberpunk a noi coeva, ma è stata anche oggetto di perlustrazioni bodyartistiche di ampio respiro, tra le quali vanno sicuramente menzionate quelle dell'australiano Stelarc (naturalizzato cipriota come Stelios Arcadiou), per il quale la tecnologia ha rappresentato, nello sviluppo della sua indagine, il mezzo privilegiato per amplificare le possibilità dell'azione corporea. ${ }^{9}$

Il soma diviene, allora, oggetto di riprogettazione, di una sperimentazione che sembra ottenere la riprogrammazione umana fino a modificarne la struttura. Le esperienze tecnomutative di Stelarc, così come quelle di Cindy Sherman, Orlan o Jana Sterback si sviluppano in seno ad una visione postumana che, pur basandosi principalmente sulla ridefinizione sociale del ruolo del corpo, lascia contemporaneamente intravedere il medesimo senso del perdersi identitario e di alterazione della forma che il tema della metamorfosi ha mosso ed animato per secoli. ${ }^{10}$ Così scrive Teresa Macrì parlando del corpo postorganico della cybercultura: «Il corpo in costruzione è un'ibridazione fantastica tra organico e inorganico, tra materia particellare e chip al silicio. Quello che il presente ci prospetta è un corpo dalle contaminazioni molteplici e dalle funzionalità imprevedibili. Queste alterazioni, a cui il corpo va incontro, spodestano la sua identità e ridefiniscono una soggettività mutante» (1996: 10). Sarà precisamente nell'ottica di uno spodestamento identitario e di un soggetto mutante, come vedremo, che funzioneranno i corpi macchinici messi in piedi dalla compagnia cesenate.

Se le nuove avanguardie artistiche, partendo dalla Bodyart degli anni Settanta fino ai contemporanei sperimentatori postorganici e cyberartisti, hanno sperimentato nell'ottica della deformazione e della liberazione organi-

9 Le vere e proprie pratiche di ibridazione tecnologica iniziano con gli esperimenti della «terza mano» (iniziati nel 1984), in cui la struttura e la percezione corporea si amplificano attraverso l'innesto di una protesi che si interfaccia all'umano. Se nelle precedenti performance delle «sospensioni» si faceva riferimento ad un corpo attraversato dal tecnologico (come se l'ergersi nel vuoto consentisse una sua smaterializzazione), ora il corpo si contamina, si duplica nel valore accrescitivo.

10 Per uno studio sistematico sul tema delle metamorfosi rimandiamo a Fusillo (2007: 1493-1497). 
ca, è con il postmoderno che il corpo si definisce compiutamente come scatola vuota, passibile di accrescimenti o amputazioni. Il corpo sarebbe qui segnato da una dissoluzione che è conseguente alla mancanza identitaria: si mutila o si accresce fondendosi con ciò che gli è alieno, diventa corpo mutante che «non riconosce la linearità di una evoluzione di specie» ma al contrario si dirige verso un'identità che si muove per sottrazione rispetto ai canoni di genere sessuale, razza o religione (Alfano Miglietti, 1997: 11). «Il corpo diviene linguaggio assoluto, medium attraverso il quale l'artista si trasforma, trasforma la propria immagine e la propria identità. Il corpo diviene materiale plasmabile» (ivi: 25).

Le ricerche performative menzionate e, con esse, quelle di Castellucci, sembrano muoversi sul doppio binario di un corpo senza organi di segno artaudiano e di un corpo accresciuto, sperimentando la genesi di nuove forme di umanità. Lo dimostra l'esperimento scenico dell'uomo a grandezza naturale, ricoperto da pelle di maiale, del gruppo catalano La Fura dels Baus (in Joan l'hombre de carne), dove si dà vita a un corpo inquietante, diviso tra splatter e fantasmatico, con un'anima cyborg che «porta a quelle zone liminari che legano vita e non vita, uomo e macchina, corpo e cervello» (Macrì, 1996: 38).

Il corpo contemporaneo, allora, è divenuto tecnocorpo dalla natura ibrida, secondo quella funzionalità protesica della macchina che già avevano evidenziato Balla e Depero nel Manifesto della ricostruzione futurista dell'universo (1915) e che esploderà con il CyborManifesto di Donna Haraway (1985). La questione dell'uomo-macchina risulterebbe quindi ormai ampiamente determinata dall'inevitabile mutazione corporea e dall'espansione tecnologica che fa dell'essere umano un soggetto in continua trasformazione: come nota Formenti, donare un corpo ad una macchina è un'offerta sacrificale: la macchina riceve un corpo dall'uomo, ma ricambia donando all'uomo i propri sensi artificiali (2003: 121).

Delineato brevemente il campo, rimane da chiedersi in che senso funzionino gli addizionamenti macchinici negli spettacoli della Raffaello Sanzio, e come sviluppino il tema del fantastico in seno al postorganico. Abbiamo notato come la performance degli anni Novanta si sia affermata come un dislocamento della corporeità che, attraversando una dimensione inorganica, ha potuto portare a termine un processo di manipolazione e alterazione del sé. Nello spettacolo Masoch Castellucci propone una scena che è minacciosa scatola di ferro, dove il protagonista, lo scrittore austriaco Leopold von Sacher Masoch, sarà oggetto di seduzioni e torture e dalla quale pendono gli strumenti della perversione (ganci e carrucole) che daranno spettacolo del marti- 
rio masochista, ritmato dal suono di stantuffi che ci riportano al rituale macchinico che si sta compiendo. È qui, più che altrove, che si celebra la simbiosi tra l'inumano ed il corpo ${ }^{11}$ dell'attore in un'alterità inassumibile e vergogno$\mathrm{sa}^{12}$ in una spettacolarità che sembra ampiamente debitrice delle "sospensioni» di Stelarc. ${ }^{13}$ Nella scena finale vedremo Leopold denudato, con delle pinze applicate sui capezzoli, un cavetto tra i denti nella bocca spalancata da un allargascarpe mentre viene appeso con tre cinghie e sollevato sopra il palcoscenico (cfr. Castellucci, 2001: 65-66). L'esplicita connotazione sadomasochista, però, trasforma la riduzione del corpo ad oggetto facendone una figurazione funzionale alla rappresentazione di una perversione più che in un'apertura vera e propria ai territori del fantastico. Diversamente accadrebbe nell'Orestea (una commedia organica?), come nel caso dei conigli meccanici che entrano in scena nel prologo guidati dal Coniglio Corifeo (chiaro rimando a Carroll) per poi esplodere o, soprattutto, per il braccio metallico che Pilade manovra dopo averlo impiantato sul corpo dell'amico Oreste. Questa scena, che doppia l'innesto tragos-Oreste che l'ha preceduta ci sembra sintetizzare perfettamente la possibilità di una fantasmagoria scenica dell'uomo-macchina capace di veicolare una pluralità ambigua di significati e dunque di innestare, attraverso l'accoppiamento incongruo, una catena di associazioni ramificate. Si visualizza qui la disseminazione del corpo e dell'identità (che James Ballard ben descrive nel suo La mostra delle atrocità) senza portare in scena la pedissequa rappresentazione del testo, quanto traducendolo in una scrittura scenica che è rovesciamento dell'interno sull'esterno ed insieme equazione postmoderna fra bellezza ed oscenità.

11 Così sostiene Castellucci parlando di Masoch: «La carne rimanda al fuori come il corpo al dentro: è il punto e il margine in cui il corpo non è più solo corpo, ma anche il suo rovescio e il suo fondo sfondato, come aveva, a suo modo, intuito Merleau-Ponty. Da qui la potenza comunitaria della figura dell'incarnazione rispetto a quella, immunitaria, dell'incorporazione o della corporazione (tipica, non a caso, di tutti i fascismi): nell'incarnazione Cristo fuoriesce dalla sua natura divina per farsi altro da sé. Direi che sia appunto la metafora dell'incarnazione l'elemento che spiega la perdurante vitalità del cristianesimo alla fine della religione cristiana. L'elemento che resiste alla sua stessa autodecostruzione perché tocca nella maniera più profonda e originaria la questione del munus comune: noi stessi come l'infinita "carne del mondo"» (Castellucci, 2001: 73).

12 Che è poi quella dell'attore. "C'è un esser-gioia per l'attore, un piacere per il masochista, che passa solo dopo aver attraversato la soglia del dolore, e questo attraversamento ha tutta la consistenza di una punizione [...] La punizione, dunque, corrisponde, nella fantasia masochista, al momento dell'esposizione della sofferenza quanto ormai essa risulti irreparabile». (Castellucci, 2001: 69) 13 Superando i masochismi espliciti degli Azionisti Viennesi degli anni Sessanta-Settanta che strutturavano le proprie performances attorno a violente mutilazioni corporee autolesioniste, le sospensioni di Stelarc, che furono oggetto della sua azione artistica a principio degli anni Settanta e in cui sospendeva in aria il suo corpo prima retto da imbragature poi da ganci infilzati alla pelle, sono da intendersi come un'educazione del corpo alla resistenza e al superamento delle condizioni limite, analogamente a quanto accade in molte forme di ritualità orientale o amerindia. 
Ribadiamo, come abbiamo più volte avuto occasione di notare, che qui non opera una riscrittura dell'opera eschilea, trattandosi di un intertesto che mette a reagire il mito (svuotato del suo fondamento politico) con i famosi racconti di Lewis Carroll, Alice nel paese delle meraviglie ed Alice dietro lo specchio. «Aldilà dello specchio ho trovato Antonin Artaud senza volerlo e senza cercarlo, quindi fuori dall'accademia [...] Da Eschilo a Carroll, da Carroll ad Artaud, e da Artaud al silenzio» (Castellucci, 2001: 157).

Già dal principio dell'opera lo spettatore è catapultato in un clima di irrealtà paradossale, che sovrappone il racconto alla fiaba, e ciò significa l'irrealizzabile tragico degli adulti con il fantastico mondo infantile. Come nella famosa favola di Alice, il Coniglio Corifeo dalla voce acuta di castrato cerca il suo orologio da taschino, gridando: «È tardi!... Oh... Com'è tardi!» (Castellucci, 2001: 97).

Già abbiamo visto come funzioni secondo le leggi del fantastico anche l'introduzione dei coniglietti di gesso, che mette in risalto contemporaneamente il rimando ad Alice e il fantasmatico dell'inorganico.

«Alice=Ifigenia. La favola di due bambine rapite in una verticalità, nel segno degli animali, nell'antimondo della parola che ora solo lui, il Coniglio, sembra poter restituire. Due favole iniziatiche in un unico destino. Solo la voce sacrificale della lepre di Ifigenia, o del coniglio di Alice, può sostenere il peso della favola tragica» (Castellucci, 2001: 116).

Il secondo atto di Orestea (una commedia organica?), Le Coefore, si apre in uno scenario bianco con il suolo completamente cosparso di farina, diviso in due parti speculari da un telo, anch'esso bianco. Entrano Pilade ed Oreste, nudi ed imbiaccati, seguiti da una Elettra obesa in tutù rosa ed un piccolo membro maschile che le penzola sull'inguine. Si apre la tomba parallelepipeda che occupa la scena (insieme ad una sedia che non smetterà mai di girare), dalla quale esce una capra morta con la testa incappucciata di bianco: è il cadavere di Agamennone. Il capro viene issato e collegato attraverso dei tubi ad Oreste, riprendendo una respirazione macchinica che lo trasforma, contemporaneamente, in innesto e doppio speculare. La scena seguente complica il rituale con l'installazione di un braccio meccanico sul corpo dell'eroe, il cui rumore si somma al respiro del caprone. L'effetto spiazzante della creazione del novello cyborg è accentuata dal cappello conico di latex di Pilade e dal naso da clown di Oreste. Sarà attraverso il braccio meccanico che Oreste ucciderà la madre, quasi che l'impianto macchinico abbia generato quell'addizione di sensi artificiali di cui parlavamo in precedenza servendoci di Formenti. 
«Il meccanismo del braccio provoca un rumore di sfiato d'aria che si somma per un poco a quello del respiro della capra. È evidente la continuità e il legame tra Oreste e Agamennone-capra, infatti avviene ora un cambio di testimone, perché il movimento del braccio armato subentra a quello del meccanismo della capra» (Castellucci, 2001: 133).

La scena appena descritta, quindi, attiva tre topoi della narratività fantastica: da una parte il doppio (doppie sono le fisionomie di Pilade e Oreste così come doppio è il nuovo organismo generato), dell'ibrido (risultante dell'addizionamento del padre sul figlio) e del cyborg (Oreste come corpo-macchina). Il continuo scivolamento nell'incertezza tra animato ed inanimato sembra in un certo senso anticipato dall'inquietante movimento ossessivo della sedia girevole, che ricorda inoltre quello specifico valore formale di molta narrativa fantastica che accumula dettagli che «fanno realtà» per poi innestarvi l'esposizione di un evento sovrannaturale (come notava Ceserani a proposito de I pomeriggi del sabato di Tabucchi, 1996: 40). Tutta la rappresentazione sembra potersi leggere come un viaggio allucinatorio di cui il protagonista è più o meno consapevole (pensiamo alla scena finale con Oreste che fissa enigmaticamente la sala, rinchiuso nella gabbia di scimmie), inscritto in un tempo dilatato e distorto.

In più, il senso dell'innesto macchinico assume un valore aggiunto se calato all'interno di una poetica del corpo, come quella che sviluppa la Societas, che ne fa un sito abietto e produttore di rifiuti e di umori, la cui esibizione sembra ricercare un primordiale non ancora articolato e declinato. Il sacrificio del corpo (che è dell'attore e del personaggio, abbiamo visto) è declinabile contemporaneamente in due usi: quello che ne fa oggetto sacrale e olocausto del sé offerto alla divinità ma anche richiamo alla solidarietà metamorfica dell'attore con l'animale, che evoca ambientazioni da mattatoio. Sintomatico è in questo senso il processo dell'eviscerazione, che pure ritorna ricorrentemente nelle prassi sceniche della Socìetas, e di cui è emblematica una scena di Genesi, ambientata ad Auschwitz, in cui una bambina abbigliata da bianconiglio (un'altra volta Carroll) descrive la natura di alcune riproduzioni di organi espiantati che calano sulla scena, rivendicandone la proprietà.

Un corpo accresciuto può essere contemporaneamente anche un corpo diviso, frammentato e scandagliabile, come avviene nella perlustrazione endoscopica del Giulio Cesare quando «...vskij»,, ${ }_{1}^{4}$ interpretando il dialogo tra Fla-

14 ...vskij, personaggio d'invenzione evidentemente alieno all'opera shakespeariana è, secondo la 
vio Marullo e il Ciabattino, mostrerà le proprie corde vocali proiettate sul fondo, nella sincronica contrazione che si produce ad ogni parola pronunciata, dopo aver inserito il lungo filo della telecamera attraverso le narici.

L'esposizione corporea della Raffaello Sanzio è, allora, un'esposizione che è, come sosteneva il già citato Castellucci, effettivamente anteriore allo stadio dello specchio, un corpo al quale non è richiesta una forma compiuta, e per questo passibile di una presentazione che lo fa variamente aperto, scorticato, addizionabile, manipolabile.

Non potendo proseguire oltre nella nostra breve panoramica sulle occorrenze fantastiche del gruppo, non ci rimane che concludere notando come queste abbiano funzionato, nella doppia declinazione di organico ed inorganico, nell'ottica di un'eversione che è il frutto, principalmente, della scrittura di scena e dell'organizzazione dei codici spettacolari. È chiamando ad interagire la pluricodicità costitutiva dell'oggetto spettacolare che Castellucci e i suoi innescano, sotto i nostri occhi, lo scandalo della connivenza tra ciò che è spiegabile e ciò che non lo è, lasciandoci sconcertati in quel bilico ermeneutico che è proprio del fantastico.

Parafrasando quanto scriveva Pezzoni su Silvina Ocampo, potremmo dire che l'inclusione di modi antitetici e di ancor più antitetiche produzioni del mondo (attraverso spettacoli che sembrano descrivere minuziosamente un accadere per poi tacerne il senso ultimo), così come, e soprattutto, il proporre sensi contraddittori ed intercambiabili, «significa instaurare il significato come scandalo» (1982: 9).

Che pochi siano gli scandali di egual portata non deve, a questo punto, meravigliare.

\section{BIBLIOGRAFIA}

Albertazzi, Silvia (1993): Il punto sulla letteratura fantastica, Laterza, Bari-Roma.

Alfano-Miglietti, Francesca (1997): Identità mutanti. Dalla piega alla piaga: esseri delle contaminazioni contemporanee, Costa \& Nolan, Genova.

CaIllois, Roger (2004): Nel cuore del fantastico, Abscondita, Milano.

nostra opinione, una delle figure più enigmatiche dell'intera messa in scena, chiaro riferimento a Stanislavskij e al teatro di regia. In questo termini ne parla lo stesso Castellucci: «Si vedeva questo personaggio annunciato con "...vskij", come la coda di un animale che sta scappando, e noi riusciamo a vedere solo la coda di questo nome finale: “...vskij", lasciando aperta la possibilità di un errore di ricostruzione. Non è esattamente Stanislavskij, non si sa... Potrebbe essere Stanislavskij, ma c'è anche qualche altro maestro che finisce per “...vskij"... È un po' come l'immagine della testa di Velasquez che fa capolino nella tela Las Meninas» (Castellucci, 2001: 274). 
CAlvino, Italo (1995): Una pietra sopra. Discorsi di letteratura e società, Mondadori, Milano.

CAMPra, Rosalba (2000): Territori della finzione. Il fantastico in letteratura, Carocci, Roma. Castellucci, Romeo e Castellucci Claudia (1992): Il teatro della Societas Raffaello Sanzio. Dal teatro iconoclasta alla super-icona, Ubulibri, Milano.

CAstellucci, Romeo et al. (2001): Epopea della polvere. Il teatro della Societas Raffaello Sanzio 1992-1999. Amleto, Masoch, Orestea, Giulio Cesare, Genesi, Ubulibri, Milano.

Ceserani, Remo (1983): La narrazione fantastica, Nistri-Lischi, Pisa. (1996): Il fantastico, Il Mulino, Bologna.

Chinzari, Stefania (1995): «Oreste, eroe maschilista», L'Unità (23 gennaio), p. 12.

CoRTI, Claudia (1989): Sul discorso fantastico. La narrazione nel romanzo gotico, ETS, Pisa.

DERRIDA, Jacques (1971): La Scrittura e la differenza, Einaudi, Torino.

Dìaz Brown, Hélène (1996): L'effet fantastique ou la mise en jeu du sujet, Anma Libri, Stanford.

Doležel, Lubomír (1999): Heterocosmica. Fiction e mondi possibili, Bompiani, Milano.

Eco, Umberto (2002): Lector in fabula. La cooperazione interpretativa nei testi narrativi, Bompiani, Milano.

Formenti, Carlo (2003): Incantati dalla rete, Raffaello Cortina, Milano.

Fusillo, Massimo (2007): «Metamorfosi», in Remo Ceserani et al. (ed.), Dizionario dei temi letterari, vol. II, Utet, Torino, pp. 1493-1497.

JACKsON, Rosemary (1986): Il fantastico. La letteratura della trasgressione, Pironti, Napoli.

KANTOR, Tadeusz (2000): Il teatro della morte. Materiali raccolti da Denis Balbet, Ubulibri, Milano.

LugnANI, Lucio (1983): «Per una delimitazione del genere», in Aa.Vv., La narrazione fantastica, Nistri-Lischi, Pisa.

LUPERINI, Romano (1990): L'allegoria del moderno. Saggi sull'allegorismo come forma artistica del moderno e come metodo di conoscenza, Editori Riuniti, Roma.

MACRì, Teresa (1996): Il corpo postorganico, Costa \& Nolan, Genova.

Mango, Lorenzo (2003): La scrittura scenica, Bulzoni, Roma.

MARChiori, Fernando (2010): Negli occhi delle bestie. Visioni e movenze animali nel teatro della scrittura, Carocci, Roma.

Pezzoni, Enrique (1982): «Prefazione a Silvina Ocampo», in Silvina Ocampo, La furia y otros cuentos, Madrid, Alianza, pp. 3-19.

Ponte di Pino, Oliviero (2013): Romeo Castellucci \& Societas Raffaello Sanzio, Doppiozero, Milano.

PrANDI, Michele, (1990): «Una figura testuale del silenzio: la reticenza», in Maria-Elisabeth Conte, Anna Giacalone Ramat, Paolo Ramat (ed.), Dimensioni della linguistica, FrancoAngeli, Milano, pp. 220-238.

Robbe-Grillet, Alain (1965): Il nouveau roman, Sugar, Milano.

SCARSELla, Alessandro (1986): «Profilo delle poetiche del fantastico», La rassegna della letteratura italiana, VIII, XC, pp. 201-220.

Solmi, Sergio (1978), «Appunti sulla letteratura fantastica», in Id., Saggi sul fantastico. Dall'antichità alle prospettive del futuro, Einaudi, Torino, pp. 126-140.

Todorov, Tzvetan (1985): La letteratura fantastica, Garzanti, Milano. 
TorTONESE, Paolo (2002): «La follia tra miracolo e prova: la terza soluzione del fantastico», in Michela Vanon Alliata (ed.), Desiderio e trasgressione nella letteratura fantastica, Marsilio, Venezia, pp. 177-185.

VAтtimo, Gianni (1983): Il pensiero debole, Feltrinelli, Milano. 